\title{
A COMPARATIVE STUDY OF BULLOUS PEMPHIGOID-180 AND 230 ANTIBODIES IN SERUM AND BLISTER FLUID SAMPLES OF PATIENTS WITH BULLOUS PEMPHIGOID
}

\author{
K. Drenovska, M. Shahid, S. Vassileva \\ Department of Dermatology and Venereology, Medical University, Sofia, Bulgaria
}

\section{BACKGROUND \& OBJECTIVE}

Bullous pemphigoid (BP) belongs to the group of subepidermal autoimmune bullous dermatoses (AIBDs) mediated by autoantibodies directed against distinct components of the cutaneous basement membrane zone (BMZ), which causes formation of blisters on the skin and/or mucous membranes. The laboratory diagnosis of BP relies on the detection of tissue-bound and serum IgG anti-BMZ autoantibodies against BP antigen 180 (BP180) and 230 (BP230). Presence of anti-BMZ antibodies has also been reported in the blister fluid of BP patients using indirect immunofluorescence (IF) microscopy on monkey esophagus and salt-split skin substrates. ${ }^{1,2}$ Although the titers of anti-BMZ antibodies in blister fluid and serum were comparable, only scarce data exist on the detection of antibodies to BP180 and BP230 using the more sensitive and specific enzyme linked immunosorbent assays (ELISA). ${ }^{3}$ The aim of the present study was to investigate whether blister fluid can be an alternative to sera for detecting anti-BP180 and anti-BP230 in the routine diagnosis of BP.

\section{METHODOLOGY}

\section{Patients}

Within a 18-month period (Dec'2015 - May'2017), 38 patients with BP attending the Department of Dermatology, University Hospital “Alexandrovska”, Sofia, Bulgaria, were studied. These were 19 males and 19 females (mean age 75.7 years, median 79, range 40 - 92). The diagnosis was based on clinical features, routine histology, direct IF microscopy showing linear deposits of IgG and/or C3 at the BMZ, and ELISA.

\section{Serum and blister fluid samples}

Simultaneous serum and blister fluid samples were collected from the patients before initiation of any systemic immunosuppressive treatment. Blister fluids were collected by syringe aspiration of intact fresh blisters. Serum and blister fluid samples were stored in aliquots at $-70^{\circ} \mathrm{C}$ until used for ELISA. Autoantibody specificity was determined in all collected samples of the 38 patients using BP180 NC16A and BP230 ELISA kits (Euroimmun).

\section{Statistical analysis}

Row data were processed with the statistical package IBM SPSS Statistics 23.0. The significance level for rejecting the null hypothesis was chosen to be $p<0.05$. Alternative analysis to compare relative proportions, criteria for validation of alternative diagnostic tests, and Cohen's Kappa coefficient were used as statistical methods.

\section{RESULTS}

Of the 38 sample pairs studied, 36 showed full correlation of the qualitative results (positive/ negative) for the presence of anti-BP180 antibodies, the latter being positive (cut-off value $20 \mathrm{RU} / \mathrm{ml}$ ) in 30 patients and negative in 6 patients (Table 1). For BP230, 25 pairs of samples showed full coincidence of the results being positive (cut-off $20 \mathrm{RU} / \mathrm{ml}$ ) or negative in 11 and 24 pairs, respectively.

Table 1. Correlation of results for ELISA BP180 and BP230 in serum and blister fluid samples.

\begin{tabular}{|c|c|c|c|}
\hline $\begin{array}{c}\text { Full } \\
\text { correlation }\end{array}$ & Positive & Negative & Total \\
\hline BP180 & 30 & 6 & 36 \\
\hline BP230 & 11 & 24 & 35 \\
\hline
\end{tabular}

$B F$, blister fluid

Positive results for anti-BP180 antibodies were detected in 32 sera and 30 blister fluid samples ( $\mathrm{n}=38$, Cohen's $\mathrm{k}=0.826)$ which represents a very good correlation of the results. ${ }^{4}$ All positive blister fluid samples corresponded to positive serum samples. Two patients were negative for BP180 in the blister fluid, while their serum samples were positive. Therefore, analysing BP180 in blister fluid instead of serum does not affect the specificity and PPV (100\%) but affects sensitivity (94\%) and mostly, the NPV (75\%) (Table 2, Fig. 1). The loss of accuracy is $5 \%$.

Table 2. Values of the validation criteria when testing ELISA BP180 and BP230 in blister fluid instead of serum.

\begin{tabular}{|c|c|c|c|c|c|}
\hline $\begin{array}{c}\text { Antibodies } \\
\text { in BF }\end{array}$ & $\begin{array}{c}\text { Sensitivity } \\
(\%)\end{array}$ & $\begin{array}{c}\text { Specificity } \\
(\%)\end{array}$ & $\begin{array}{c}\text { PPV } \\
(\%)\end{array}$ & $\begin{array}{c}\text { NPV } \\
(\%)\end{array}$ & $\begin{array}{c}\text { Accuracy } \\
(\%)\end{array}$ \\
\hline BP180 & 94 & 100 & 100 & 75 & 95 \\
\hline BP230 & 85 & 96 & 92 & 92 & 92 \\
\hline
\end{tabular}

$B F$, blister fluid; $N P V$, negative predictive value; $P P V$, positive predictive value

Similarly, anti-BP230 antibodies were positive in the serum and blister fluid of 13 and 12 patients, respectively $(\mathrm{n}=38$, Cohen's $\mathrm{k}=0.821)$. In addition, in 17 out of 38 patients $(44.7 \%)$ there was full agreement between ELISA BP180 and BP230 results in the serumblister fluid pairs, all positive in 12 or all negative in 5 patients, respectively. In the remaining 21 patients the blister fluid was positive only for anti-BP180 known to be of major pathogenic importance in BP. Only 3 out of 38 blister fluid samples showed negative results for both autoantibodies, whereas the corresponding serum samples were positive either for BP180 or BP230 antibodies. The search of BP230 in blister fluid instead of serum affects to a lesser extent the test specificity (96\%), but to a higher one the PPV and NPV

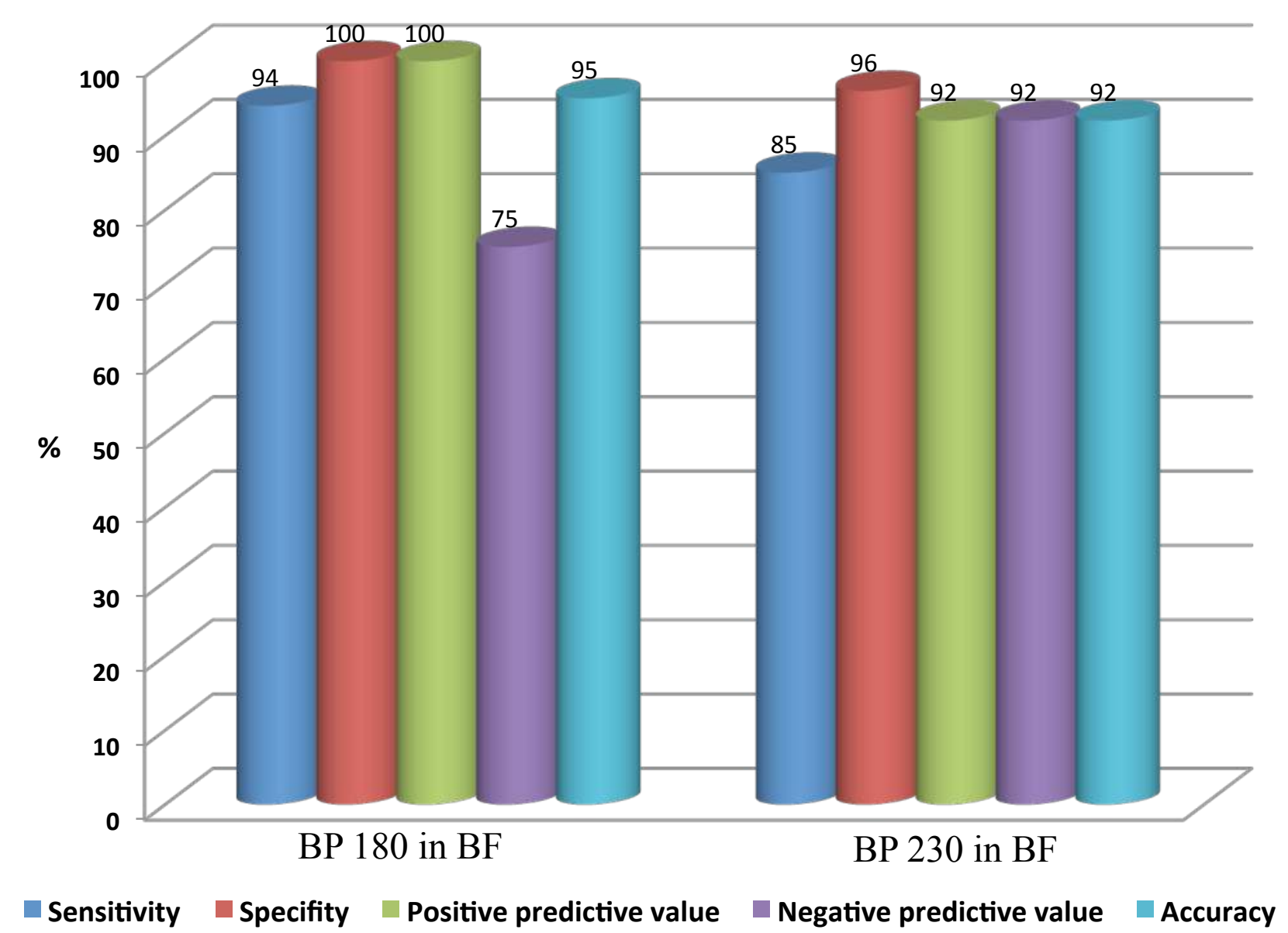
( $92 \%$ for both), and mostly the sensitivity ( $85 \%$ ) (Table 2), with $8 \%$ loss of accuracy.

Fig. 1. Values of the validation criteria for BP180 and BP230 antibodies in blister fluid instead of serum ( $B F$, blister fluid).

\section{CONCLUSION}

Our results demonstrate a relatively high agreement between the anti-BP180 and anti-BP230 values in the serum-blister fluid pairs, the latter being less invasive and easier to obtain in the routine diagnosis of BP, especially in elderly patients or children.

\section{REFERENCES}

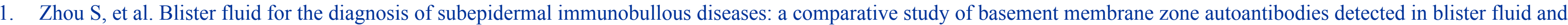
serum. Br J Dermatol. 1998; 139:27-32.

2. Daneshpazhooh M, et al. A comparative study of antibody titers of blister fluid and serum in patients with subepidermal immunobullous diseases. Int J Dermatol. 2004; 43:348-51

3. Patsatsi A, et al. Detection of anti-BP180NC16a and anti-BP230 autoantibodies in blister fluid of patients with bullous pemphigoid: the first survey in Greece. Clin Exp Dermatol. 2007; 33:183-5. 